\title{
A Note on Reinforcement of Polymer Matrix Composites Using Carbon Residues Derived From Woody Biomass
}

\author{
Malte Pries AND Holger Militz \\ Department of Woodbiology \& Woodproducts, University of Göttingen \\ Göttingen 37077, Germany \\ BARry GOODEll, Xinfeng XIE* AND Yuhui QIAN \\ Wood Science and Technology, University of Maine, Orono, ME 04469, USA \\ Michael Peterson \\ Department of Mechanical Engineering, University of Maine, Orono, ME 04469, USA \\ ROBERTO LOPEZ-ANIDO \\ Department of Civil and Environmental Engineering, University of Maine \\ Orono, ME 04469, USA
}

\begin{abstract}
The mechanical properties of phenolic resin reinforced with three different carbon materials were investigated experimentally. The carbon materials: (1) commercially produced carbon nanotubes (CNTs), (2) flash-heated lignocellulose containing CNTs and carbon-black, and (3) cyclically oxidized lignocellulose (Goodell, B. et al. (2008). Journal of Nanoscience and Nanotechnology, 8: 2472-2474) were added to phenolic resin in different weight percentages to fabricate composites. Carbon nanotubes were found to be an effective reinforcing filler increasing tensile strength by $45.34 \%$ and Young's modulus by $19.08 \%$ with a $2 \%$ loading. The flash-heated material increased Young's modulus by $11.04 \%$ with a $2 \%$ loading but did not affect tensile strength. The cyclically heated material did not contain CNTs, their inclusion in the composites reduced Young's modulus and, for the $1 \%$ loading, reduced tensile strength as well.
\end{abstract}

KEY WORDS: carbon materials, nanotubes, woody biomass, polymer composites, phenolic composites, tensile test, strength enhancement.

\section{INTRODUCTION}

$\mathbf{F}$ OLLOWING THE VISUALIZATION of carbon nanotubes (CNTs) by Radushlevich and Lukyanovich [1] and the later improved understanding of these materials by Iijima [2],

\footnotetext{
*Author to whom correspondence should be addressed. E-mail: xinfeng.xie@umit.maine.edu 
a great interest has been shown by researchers in the field because of the excellent mechanical and electrical properties of CNTs. Carbon nanotubes are made up of carboncarbon single bonds, which is the strongest of all bonds [3]. In 1996, Young's modulus for CNTs was estimated to be as high as $1.8 \mathrm{TPa}$ [4]. More recent work has adjusted this result downwards. Demczyk et al. (2002) found a Young's modulus of 0.9 TPa and a tensile strength of $0.15 \mathrm{TPa}$ [5]. Peng et al. [6] found similar values. Even with this wide variation, the mechanical properties of CNTs are superior to any other material. In addition to their mechanical properties, CNTs also show interesting electrical properties. They can conduct electricity longitudinally, but simultaneously function as insulators in the lateral direction [7].

Due to their excellent properties, CNTs have been studied as reinforcing filler materials in a wide variety of composite materials. With relatively small additions of CNTs, large increases in mechanical properties have been observed in previous studies [8-10]. However, the reinforcement of polymers with CNTs raises several problems; the most important of which is that theoretically possible properties due to reinforcement of materials have not been obtained in practice. These discrepancies are explained in several ways.

In some cases, inconsistent or weak CNT-matrix bonding is apparent, and the CNTs are pulled out of the matrix during crack propagation [11]. This has an influence on the maximum tensile strength but it has only a limited effect on Young's modulus for small elastic deformation [12]. The greatest influence on Young's modulus is seen in the CNT diameter; a large diameter resulting in a lower Young's modulus than a small diameter for a given mass fraction of CNTs [12]. Load is transferred to the CNTs through shear stresses at the CNT-matrix interface. The layers of multi-walled CNTs are only connected by weak van der Waals forces. As a consequence of this fact, the outer layer of the multi-walled CNTs will carry almost the entire load transferred to the CNT thus resulting in reduced strength [11].

Lack of adequate dispersion also limits the functional application of CNTs as, even if successfully dispersed, agglomeration will reoccur. Several approaches have been used to disperse CNTs in a matrix, including ultrasonication, shear mixing or bonding of other substances to the surface of the CNT, none of which have worked satisfactorily. Even when it has been possible to disperse CNTs, it has not been possible to control the arrangement in the matrix. To maximize reinforcement, the nanotubes must be arranged parallel to the axis in which stresses occur [13]. No successful techniques have been created to arrange the nanotubes properly and on a large scale.

Two recent studies have shown that it might be possible to produce CNTs from biomass. Kang et al. [14] produced CNTs from grass; Goodell et al. [15] reported the production of CNTs from wood with a similar process. Besides the low temperatures necessary for these processes, the use of carbon residues and CNTs produced from wood and plant fiber may provide additional advantages over the use of conventional nanoscale carbon materials. For example, CNTs can theoretically be produced in a pre-aligned template using the wood fiber as the template material [16]. The carbonized fiber template could then be aligned with other fibers more readily in a composite preform infused with resin to address bonding and dispersion issues discussed above and discussed previously by Goodell et al. [15]. The objective of this study was, to introduce carbonized material obtained by the cyclic heating process described by Goodell et al. [15] and a flash-heating process into cast phenolic resin to test the ability of these materials to 
reinforce structural properties of polymer composites. It was known that both processes will result only in limited production of CNTs, but the question was, if even these small amounts of CNTs might result in a useful reinforcement. The idea behind this was that, due to the different structure and the potentially parallel arrangement of the produced CNTs the reinforcement might be somewhat different from the reinforcement with commercial CNTs. The ability of these materials to aid in composite reinforcement was compared to that of commercial CNTs.

\section{MATERIALS AND METHODS}

Three carbon materials were tested for reinforcement of cast polymer matrix composite samples: Commercially produced CNTs, cyclically heated thermo-mechanical pulp (TMP) fibers and flash-heated pine needles.

The multi-walled CNTs were purchased from Sigma Aldrich (Product Number 677248). They were produced via a carbon vapor deposition process by Arcema Inc. The outer diameter ranged from 10 to $15 \mathrm{~nm}$ and the length from 0.1 to $10 \mu \mathrm{m}$. The CNT content was $>90 \%$; the material contained no amorphous carbon impurities [17].

Cyclically heated material was produced using an oxidation process described in Goodell et al. [15]. Briefly, TMP fiber (Pinus ponderosa Doug. ex Laws.) was heated in air at a rate of $5^{\circ} \mathrm{C} / \mathrm{min}$ to a maximum of $110^{\circ} \mathrm{C}$. This temperature was held for $60 \mathrm{~min}$ and then was increased at a rate of $1.5^{\circ} \mathrm{C} / \mathrm{min}$ to $240^{\circ} \mathrm{C}$ and held there for $120 \mathrm{~min}$. After this pre-treatment, the fibers were placed in a $9 \mathrm{ml}$ porcelain boat and inserted into a $500 \mathrm{ml}$ ceramic tube. The tube was evacuated to $1.3 \mathrm{kPa}(9.7 \mathrm{~mm} \mathrm{Hg})$ and then air was introduced at a rate of $0.5 \mathrm{mg}$ air per $1 \mathrm{mg}$ fiber. The tube was rapidly heated to $400^{\circ} \mathrm{C}$ and maintained at this temperature for $10 \mathrm{~min}$ before cooling to room temperature. This cycle was repeated 35 times. The resulting material was crushed with mortar and pestle to a powder fine enough to be mixed into the resin.

The flash-heating treatment was conducted using pine (Pinus strobus L.) needles, which had previously been dried for $24 \mathrm{~h}$ at $105^{\circ} \mathrm{C}$. The needles were then dipped in a $15 \%$ solution of nickelchloride-hexahydrate $\left(15 \% \quad \mathrm{NiCl}_{2} \cdot 6 \mathrm{H}_{2} \mathrm{O}\right)$ and dried again for $12 \mathrm{~h}$. After this pre-treatment, approximately $30-40 \mathrm{mg}$ of the needle material was rapidly batch heated in a test tube at $1100^{\circ} \mathrm{C}$. The test tube was first heated in a tube furnace and the treated pine needles were then rapidly inserted into the tube and flash heated for $3 \mathrm{~s}$. The tube was then cooled to room temperature and the resulting material crushed with mortar and pestle to a powder fine enough to be mixed into the resin.

All carbon materials were observed under the transmission electron microscope (Phillips CM 10; operated at $100 \mathrm{kV}$ ) on carbon coated copper grids to determine the nature of the carbon and specifically to observe the presence or absence of CNTs.

A thermosetting phenolic resin 'Durite Phenolic Resin SC-1008' (Mektech Composites Inc. (New Jersey); Borden Chemicals, manufacturer) was used for the matrix. This resin has a low viscosity, which was determined to be approximately $90 \mathrm{mPa}$ s at $40^{\circ} \mathrm{C}$. Two weight percentages $(1 \%$ and $2 \%$ ) of the three carbon materials were added to the phenolic resin. The carbon materials were dispersed by ultrasonication for $30 \mathrm{~min}$ at $60^{\circ} \mathrm{C}$. After ultrasonication, the resin was poured into rectangular aluminum dishes to cast into 
$85 \mathrm{~mm}$ wide, $110 \mathrm{~mm}$ long, and $3 \mathrm{~mm}$ thick plates. The following heating schedule was used to cure the resin:

$\begin{array}{llll}0-24 \mathrm{~h} & 45^{\circ} & 78-90 \mathrm{~h} & 120^{\circ} \\ 24-48 \mathrm{~h} & 65^{\circ} & 90-98 \mathrm{~h} & 150^{\circ} \\ 48-66 \mathrm{~h} & 75^{\circ} & 98-104 \mathrm{~h} & 180^{\circ} \\ 66-78 \mathrm{~h} & 90^{\circ} & & \end{array}$

After curing, tensile test specimens were cut according to ASTM Standard 638 (sample type V) [18] using a water jet cutter (Bengal water jet; pressure:124 MPa). The mechanical properties of the materials were evaluated through a tension test (Instron $100 \mathrm{kN}$; load cell $4.4 \mathrm{kN}$ ) using a loading speed of $0.1 \mathrm{~mm} / \mathrm{min}$. Failure of the samples was achieved within 2-3 min; the longitudinal strain was measured using a non-contact digital image correlation system (ARAMIS Optical 3D Deformation Analysis; Manufacturer: GOM mbH Optical Measuring Techniques). The camera speed was adjusted to $1 \mathrm{~Hz}$. Each test datum was based on the arithmetic average of at least seven specimens.

Specimens tested included: Neat phenolic resin plates, CNT/phenolic composite plates, flash-heated/phenolic composite plates and cyclically heated/phenolic composite plates; all produced at both a $1 \%$ and $2 \%$ reinforcement level.

\section{RESULTS AND DISCUSSION}

\section{Electron Microscopy}

The commercially produced CNTs were found to be about $10-20 \mathrm{~nm}$ in diameter and several hundred $\mathrm{nm}$ in length, which conformed to the supplier's specifications. Significant amorphous carbon impurities were not observed under TEM in these CNTs (Figure 1).

The flash-heated material contained a small number of large diameter CNTs (approx. $50 \mathrm{~nm}$ ), with a length of several hundred $\mathrm{nm}$ (Figure 2). The percentage of this fraction could not be determined.

The material also contained a large fraction of carbon-black spheres of approximately $300 \mathrm{~nm}$ diameter; an example of which is shown in Figure 3. The remaining material was amorphous carbon with irregular shape and even structure.

No CNTs were observed in the cyclically heated material in this study, but the applied process showed CNT formation in an earlier work [15] reflecting the difficulty of reproducibly producing CNTs in this manner.

\section{Mechanical Properties}

The Young's modulus and the tensile strength of the pure phenolic resin were found to be $4.5 \mathrm{GPa}$ and $36.4 \mathrm{MPa}$, respectively, with a strain of 0.0087 . These control values were used to compare the mean effect of the three carbon reinforcements using a double sided $t$-test at a significance level of 0.05 .

The addition of commercial CNTs resulted in a significant increase in Young's modulus ranging from $12.1 \%$ to $19.1 \%$ compared to the control values (Figure 4). Loading the resin with flash-heated material increased Young's modulus as well, with a $1 \%$ loading 


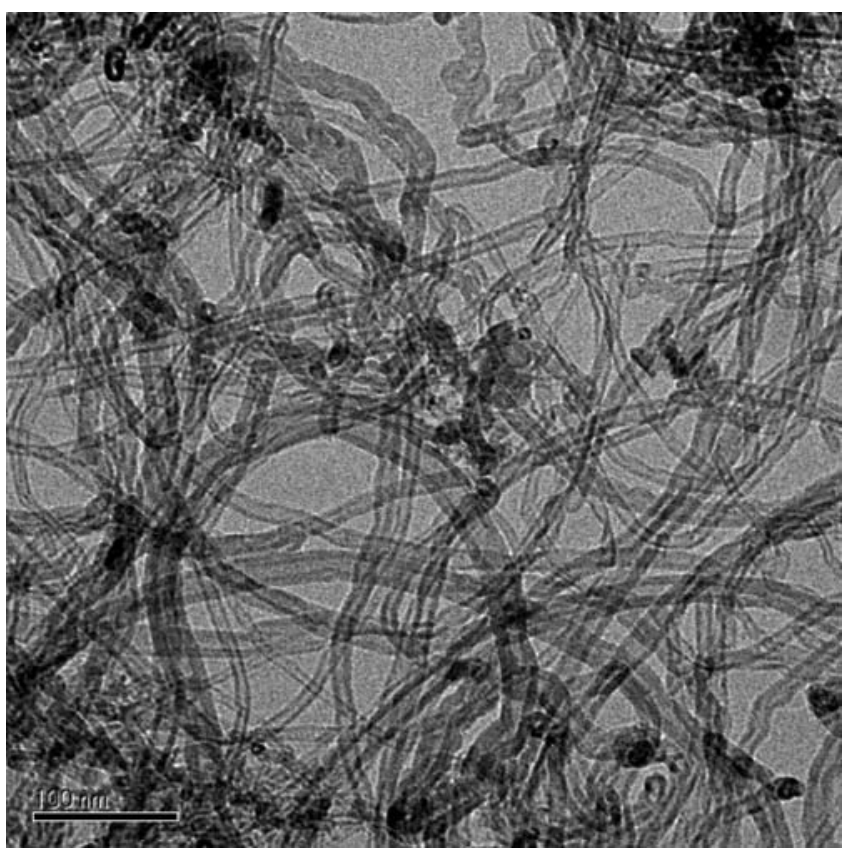

Figure 1. Carbon nanotubes used in composite reinforcement.

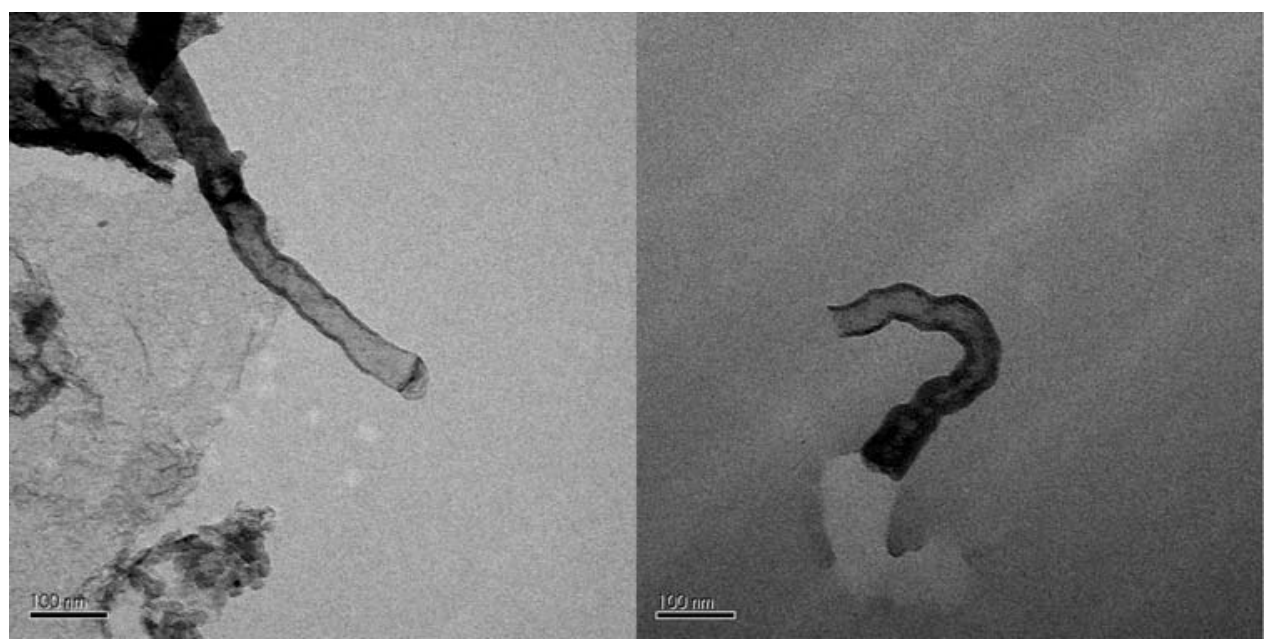

Figure 2. Carbon nanotubes in the flash-heated material.

providing a significant increase of $10.5 \%$; a value statistically the same as reinforcement with $1 \%$ CNTs. A $2 \%$ loading resulted in an $11 \%$ increase, however, which was not significantly different from the $1 \%$ loading value. The cyclically heated material reduced Young's modulus at both loading levels compared to the control with $1 \%$ and $2 \%$ loadings, reducing it by $14.8 \%$ and $13.8 \%$, respectively.

Tensile strength (Figure 5) was not significantly changed with a $1 \%$ CNT loading, but a loading of $2 \%$ CNTs increased tensile strength significantly by $45.3 \%$. The flash-heated 


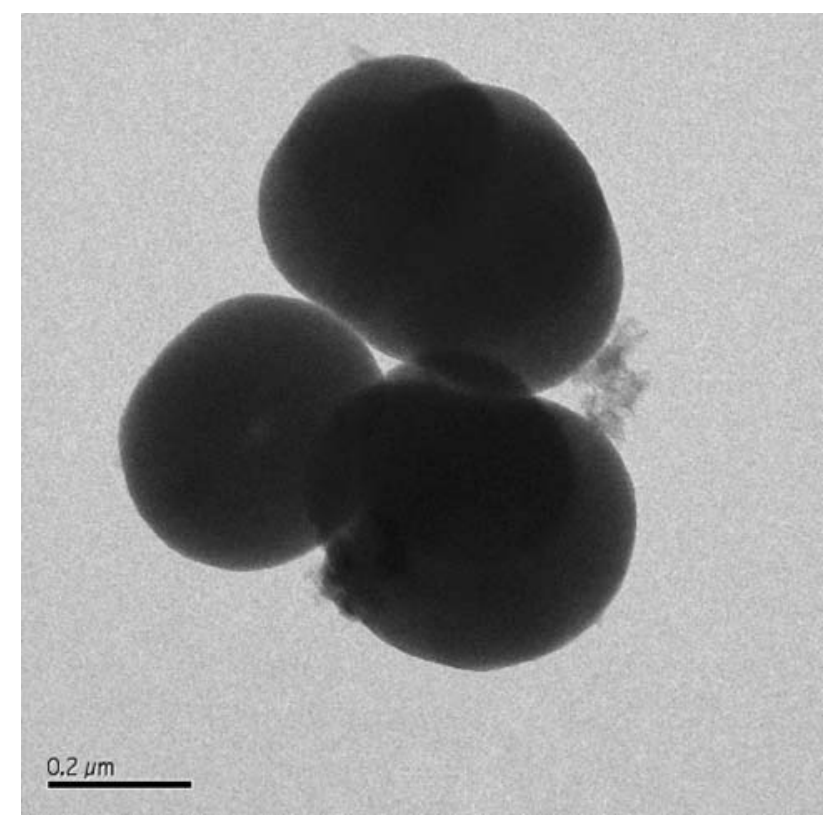

Figure 3. Carbon-black in the flash-heated material.

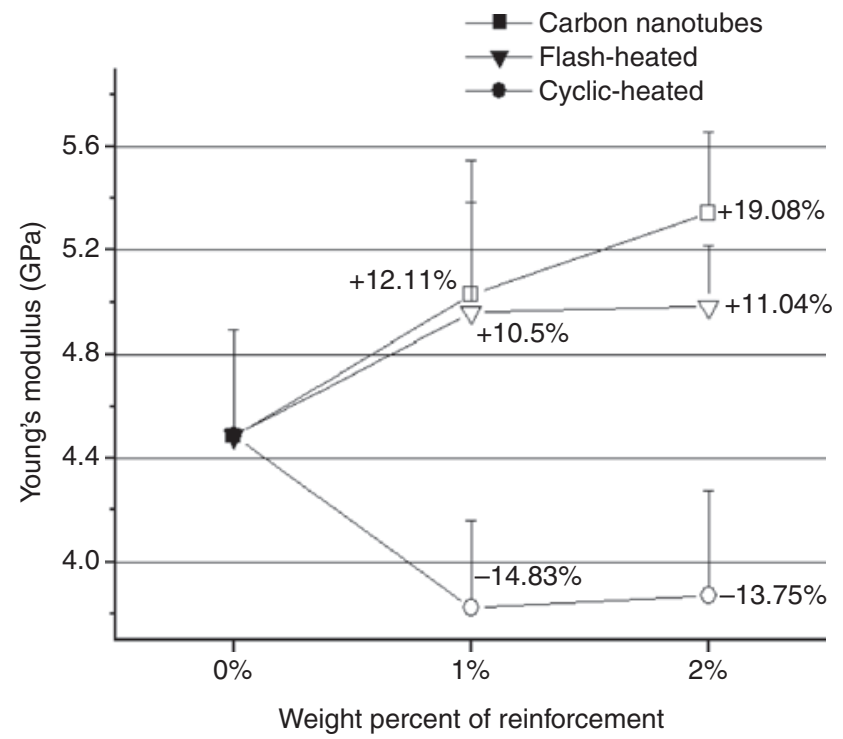

Figure 4. Young's modulus (arithmetic means) plotted against the weight percent of reinforcement of three carbon materials. Hollow (unfilled) symbols indicate that a value is significantly different compared to the control values. (The Lines are not stating continuous functions).

material loadings did not significantly affect tensile strength. The cyclically heated material reduced tensile strength with a $1 \%$ loading of the phenolic resin by $25 \%$. A $2 \%$ loading with the cyclically heated material, however, did not change tensile strength compared to control values. 


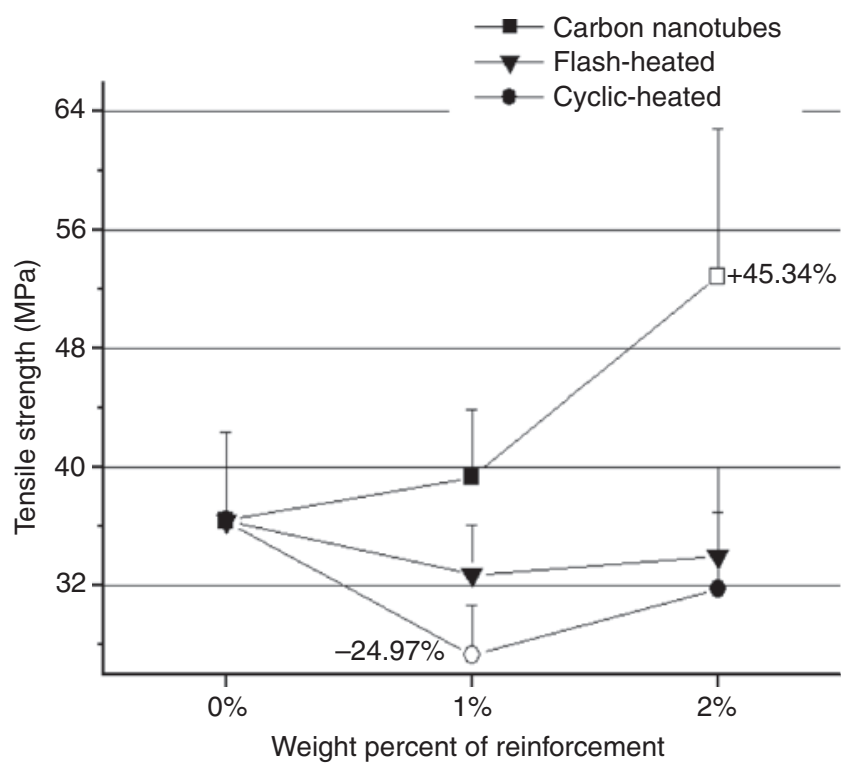

Figure 5. Tensile strength (arithmetic means) plotted against the weight percent of reinforcement of three carbon materials. Hollow (unfilled) symbols indicate that a value is significantly different compared to the control values. (The Lines are not stating continuous functions).

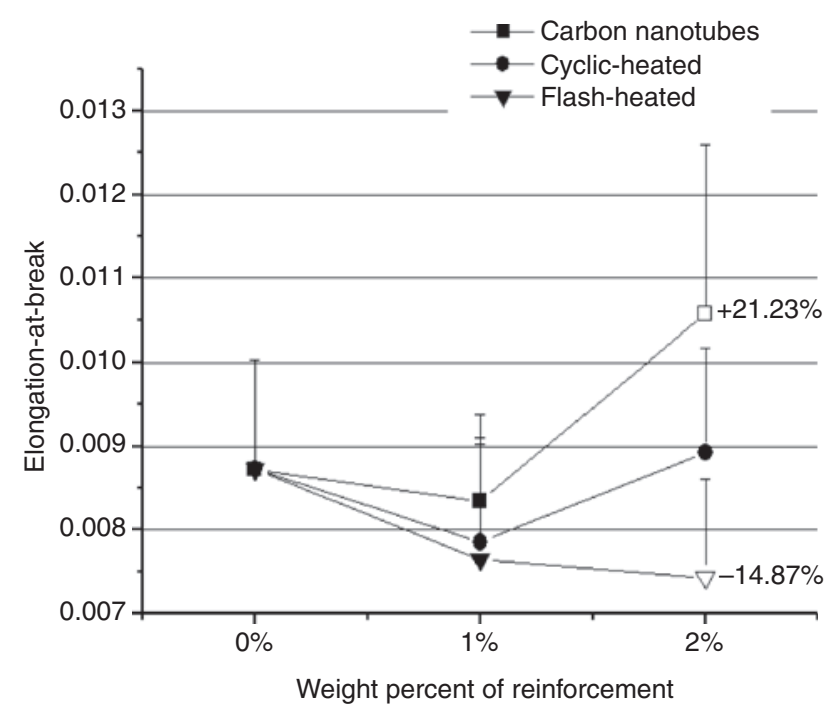

Figure 6. Elongation-at-break (arithmetic means) plotted against the weight percent of reinforcement of three carbon materials. Hollow (unfilled) symbols indicate that a value is significantly different compared to the control values. (The Lines are not stating continuous functions).

The strain (Figure 6) increased to 0.0106 with the addition of $2 \%$ CNTs; a significant increase of $21.2 \%$ over the control value. The addition of the cyclically heated material showed no significant effect on the strain at either level. The flash-heated material reduced the strain significantly with a $2 \%$ loading resulting in a $14.9 \%$ reduction. 
Carbon nanotubes have been found to enhance the properties of polymer matrix composites in earlier studies $[8,19,20]$. Yeh et al. [19] reinforced phenolic resin with CNTs and observed an increase in Young's modulus and tensile strength as the CNT ratio increased. They achieved greater reinforcement (44\% increase in Young's modulus and $64.6 \%$ increase in tensile strength with a $2 \%$ loading of CNTs) than reported in the current work, but used a different curing schedule; curing their resin in a hot press at $170^{\circ} \mathrm{C}$ for $2 \mathrm{~h}$ after a two day vacuum treatment. ${ }^{1}$

In the current research, the flash-heated material increased Young's modulus but tensile strength was not affected. Since the strain also decreased this may have implications for the toughness of the material, albeit modest. The reinforcement with CNTs on the other hand increased modulus, strength and strain, indicating an increase in toughness of the resin. The reinforcing effects of these materials were, for this reason, quite different.

Several factors may be the reason for the different behavior of the flash-heated material, for example the size of the CNTs, the different dispersion and the type of reinforcement materials. Size differences of CNTs have been shown to be a factor in previous work [12]. In our current research, the commercially produced CNTs had diameters of 10-15 nm [17] whereas the CNTs in the flash-heated material had diameters of about $50 \mathrm{~nm}$. The dispersion level was also different in the three materials. While the commercial CNTs agglomerated during curing, the two other materials remained evenly dispersed. It is unclear however, if the CNTs contained in the flash-heated material remained dispersed as even though they appeared visually to be well dispersed, some agglomeration cannot be ruled out. Another possible explanation could be that the effects of reinforcement may not be primarily attributable to the CNTs in the flash-heated material. In the flash-heated material a high fraction of carbon-black existed, which is also used as a filler material and can affect plastic matrix properties [21]. Thus the carbon-black reinforcement could account for at least some of the effects on the phenolic resin along with, or perhaps even, instead of the CNTs. However, the large number of unknown factors in the behavior of the flash-heated material makes it difficult to evaluate the mechanisms for the improvement of properties in the phenolic resin amended with the flash-heated material.

The addition of the cyclically heated material to the resin decreased the Young's modulus and, at a 1\% mixture, also decreased the tensile strength. The material did not contain any detectable CNTs or carbon-black but instead contained primarily amorphous carbon wood residue. Because carbonized wood produced at low temperatures has reduced mechanical properties [22], the carbon materials produced using cyclical heating process cannot be recommended as a promising reinforcement material for plastics.

To obtain effective reinforcement of composites with carbon materials produced from plant materials, this research suggests that it will be important to have a high yield of CNTs in the products. Unfortunately, to date no method to produce a high yield of CNTs from plant materials is known. However, the results in this work show that even low CNT contents such as those produced in the flash-heated material can potentially have an effect in aiding reinforcement to composite materials. This is especially interesting, because the total content of CNTs in the composite was much lower than in the composite with the commercially produced CNTs.

\footnotetext{
${ }^{1}$ Curing in a hot press was tested but did not work in the current study as the water produced from the condensation reaction inhibited the formation of useful resin plates. The short cure time used by Yeh et al. could also have limited agglomeration of the CNTs to produce improved reinforcement.
} 


\section{CONCLUSION}

In this study the reinforcing effect of CNTs, a cyclic heated material, and a flash-heated material produced, respectively, from wood fiber ( $P$. ponderosa) and pine needles ( $P$. strobus), on the mechanical properties of phenolic resin was investigated experimentally. The phenolic resin was reinforced with two weight percentages of these materials, $1 \%$ and $2 \%$. The following conclusions can be drawn:

1. The reinforcing effect of the CNTs was greatest, with Young's modulus and tensile strength enhanced as well as the elongation-at-break. This result was found previously $[8,19,23]$ but the effect of reinforcement was less in this study compared to others and was likely associated with the long curing time for the phenolic resin which allowed the CNTs to agglomerate in the resin.

2. The flash-heated material, which contained some CNTs and a larger fraction of carbon-black increased Young's modulus but did not affect tensile strength. The effect on material properties was therefore different compared to the effect of commercially produced CNTs. Although this effect may have been produced by the CNTs in the flash-heated material, because carbon-black was also present in the flash-heated material, a combination of the two materials may be responsible for the effect. The data did not permit a clear determination of this.

3. The cyclically heated material reduced Young's modulus and, with a $1 \%$ loading, tensile strength as well. The material did not contain CNTs (as was reported previously [15]) or carbon-black in traceable amounts but instead contained primarily amorphous carbon.

This research suggests that carbonized woody residues could potentially be used for reinforcement in polymer matrix composites, but that material containing greater percentages of either carbon-black or CNTs would enhance the reinforcement potential.

\section{ACKNOWLEDGMENTS}

This is publication 3068 of the Maine Agricultural and Forest Experiment Station. We appreciate the support of the National Wood Utilization Research (WUR) grant program for partial support of this work.

\section{REFERENCES}

1. Radushkevich, L.V. and Lukyanovich, V.M. (1952). O Strukture Ugleroda, Obrazujucegosja Pri Termiceskom Razlozenii Okisi Ugleroda Na Zeleznom Kontakte, Zurn Fisica Chimica, 26: $88-95$.

2. Iijima, S. (1991). Helical Microtubules of Graphitic Carbon, Nature, 354: 56-58.

3. Coulson, C.A. (1961). Valence, 2nd edn, Oxford University Press, London.

4. Treacy, M.J., Ebbesen, T.W. and Gibson, J.M. (1996). Exceptionally High Young's Modulus Observed for Individual Carbon Nanotubes, Nature, 381: 678-680.

5. Demczyk, B.G., Wang, Y.M., Cumings, J., Hetman, M., Han, W., Zettl, A. and Ritchie, R.O. (2002). Direct Mechanical Measurement of the Tensile Strength and Elastic Modulus of Multiwalled Carbon Nanotubes, Materials Science and Engineering A - Structural Materials Properties, 334: 173-178. 
6. Peng, B., Locasio, M., Zapol, P., Li, S.Y., Mielke, S.L., Schatz, G.C. and Espinosa, H.D. (2008). Measurements of Near-ultimate Strength for Multiwalled Carbon Nanotubes and Irradiationinduced Crosslinking Improvements, Nature Nanotechnology, 3: 626-631.

7. Popov, V.N. (2004). Carbon Nanotubes: Properties and Application, Materials Science \& Engineering $R$ - Reports, 43: 61-102.

8. Tai, N.H., Yeh, M.K. and Peng, T.H. (2008). Experimental Study and Theoretical Analysis on the Mechanical Properties of SWNT/Phenolic Composites, Composites Part B - Engineering, 39: 926-932.

9. Allaoui, A., Bai, S., Cheng, H.M. and Bai, J.B. (2002). Mechanical and Electrical Properties of a MWNT/Epoxy Composite, Composites Science and Technology, 62: 1993-1998.

10. Bai, J. (2003). Evidence of the Reinforcement Role of Chemical Vapour Deposition Multiwalled Carbon Nanotubes in a Polymer Matrix, Carbon, 41: 1325-1328.

11. Lau, K.T. and Hui, D. (2002). Effectiveness of Using Carbon Nanotubes as Nanoreinforcements for Advanced Composite Structures, Carbon, 40: 1605-1606.

12. Thostenson, E.T. and Chou, T.W. (2003). On the Elastic Properties of Carbon Nanotube-based Composites: Modelling and Characterization, Journal of Physics D: Applied Physics, 36: 573-582.

13. Mallick, P.K. (1993). Fiber-reinforced Composites: Materials, Manufacturing and Design, M. Dekker, New York.

14. Kang, Z.H., Wang, E.B., Mao, B.D., Su, Z.M., Chen, L. and Xu, L. (2005). Obtaining Carbon Nanotubes from Grass, Nanotechnology, 16: 1192-1195.

15. Goodell, B., Xie, X., Qian, Y., Daniel, G., Peterson, M. and Jellison, J. (2008). Carbon Nanotubes Produced from Natural Cellulosic Materials, Journal of Nanoscience and Nanotechnology, 8: 2472-2474.

16. Xie, X., Goodell, B., Qian, Y., Daniel, G., Zhang, D., Nagle, D., Peterson, M. and Jellison, J. (2009). A Method for Producing Carbon Nanotubes Directly from Plant Materials, Forest products Journal, 59(1/2): 26-28.

17. Sigma Aldrich (2008). Product Information. Available at: www.sigmaaldrich.com.

18. American Society for Testing and Materials (2008). Standard Test Method for Tensile Properties of Plastics, ASTM Standard D 638.

19. Yeh, M.K., Tai, N.H. and Lin, Y.J. (2008). Mechanical Properties of Phenolic-based Nanocomposites Reinforced by Multi-walled Carbon Nanotubes and Carbon Fibers, Composites Part A - Applied Science and Manufacturing, 39: 677-684.

20. Tai, N.H., Yeh, M.K. and Liu, H.H. (2004). Enhancement of the Mechanical Properties of Carbon Nanotube/Phenolic Composites Using a Carbon Nanotube Network as the Reinforcement, Carbon, 42: 2774-2777.

21. International Carbon Black Association (2008). Available at: http://carbon-black.org (accessed October 8, 2008).

22. Xie, X., Goodell, B., Qian, Y., Peterson, M. and Jellison, J. (2008). Significance of the Heating Rate on the Physical Properties of Carbonized Maple Wood, Holzforschung, 62: 591-596.

23. Yeh, M.K., Tai, N.H. and Liu, J.H. (2006). Mechanical Behavior of Phenolic-based Composites Reinforced with Multi-walled Carbon Nanotubes, Carbon, 44: 1-9. 